\title{
Geleitwort (1. Auflage)
}

Gesundheitsökonomen prognostizieren, dass die Zahl der gastroenterologischen Patienten in den nächsten 20 Jahren um $22 \%$ zunehmen wird. Dies liegt um mehr als $13 \%$ über dem Anstieg des generellen medizinischen Bedarfes und ist in erster Linie der Zunahme älterer Patienten mit gastroenterologischen Erkrankungen geschuldet. Die Deutsche Gesellschaft für Gastroenterologie, Verdauungs- und Stoffwechselkrankheiten (DGVS) hat diesen Trend früher als andere erkannt und widmet sich seit mehr als 10 Jahren mit einer eigenen Arbeitsgruppe „Geriatrische Gastroenterologie“ dieser Entwicklung. Ältere Menschen sind auf verschiedene Weise besonders in ihren medizinischen und sozialen Bedürfnissen. Dem widmet sich dieser Band, der von Werner Mayet, dem Sprecher der Arbeitsgemeinschaft Geriatrische Gastroenterologie der DGVS und seinen Kolleginnen und Kollegen verfasst wurde. Auf mehr als 400 Seiten wird auf spezifische Anforderungen in Diagnostik und Therapie geriatrischer Patienten eingegangen - soweit das Fachgebiet der Viszeralmedizin und Gastroenterologie davon berührt ist. Neben wichtigen Aspekten der Pharmakologie und der Ernährungsmedizin werden auch pathophysiologische Zusammenhänge sehr übersichtlich erklärt. Aus Sicht der Fachgesellschaft ist dieses Buch ein Meilenstein der langjährigen Aktivitäten der Arbeitsgemeinschaft „Geriatrische Gastroenterologie“ und wird auf diesem Feld Standards setzen. Der Vorstand der DGVS wünscht dem Buch eine weite Verbreitung und eine neugierige und fachkundige Leserschaft.

Prof. Dr. Markus M. Lerch

Präsident der DGVS 
